\title{
Development of Zinc Oxide Spatial Light Modulator for High-Yield Speckle Modulation
}

\author{
Naoya $_{\text {TATE }}^{\dagger \text { a) }}{ }^{\text {, Tadashi KAWAZOE }}{ }^{\dagger \dagger}$, Shunsuke NAKASHIMA ${ }^{\dagger}$, Wataru NOMURA ${ }^{\dagger}$, Nonmembers, \\ and Motoichi OHTSU ${ }^{\dagger \dagger}$, Member
}

\begin{abstract}
SUMMARY In order to realize high-yield speckle modulation, we developed a novel spatial light modulator using zinc oxide single crystal doped with nitrogen ions. The distribution of dopants was optimized to induce characteristic optical functions by applying an annealing method developed by us. The device is driven by a current in the in-plane direction, which induces magnetic fields. These fields strongly interact with the doped material, and the spatial distribution of the refractive index is correspondingly modulated via external control. Using this device, we experimentally demonstrated speckle modulation, and we discuss the quantitative superiority of our approach.

key words: nanophotonics, spatial light modulator, laser speckle, speckle modulation, oxide semiconductor
\end{abstract}

\section{Introduction}

Laser projection systems are advanced display systems with high resolution, high brightness, and good color reproducibility. The images displayed by such systems are formed by scanning a laser beam on a screen at high speed to produce an image pixel-by-pixel. However, due to the use of a laser source, observers close to the screen observe unavoidable speckle patterns [1], which drastically reduces the quality of displayed images. To further widen the adoption of of laser projection systems and make them suitable for actual environments, there is a strong demand for novel techniques that can alleviate the problems caused by speckle patterns.

Existing techniques for speckle reduction are classified into two categories: One is to apply decoherence to the laser light, and the other is to obtain a time-averaged image of modulated speckle patterns. First, because speckle patterns are the result of interference of scattered light, applying decoherence to the light source can directly reduce the speckle patterns [2]-[10]. Actually, incoherent light sources, such as solar light and lamps, do not form any speckle patterns. On the other hand, because the human eye obtains an image over a certain exposure time, speckle patterns that are modulated at sufficiently high speed are time-averaged and become uniform. In such a case, the observer cannot recognize any speckle pattern [11]-[25]. In this paper, as the

Manuscript received February 29, 2016.

Manuscript revised June 7, 2016.

${ }^{\dagger}$ The authors are with Kyushu University, Fukuoka-shi, 8190395 Japan.

${ }^{\dagger}$ The authors are with Research Institute of Nanophotonics, Tokyo, 112-0014 Japan.

a) E-mail: tate@ed.kyushu-u.ac.jp

DOI: 10.1587/transele.E99.C.1264 earliest stages of the latter approach, we exhibit a novel device, which is developed and operates with an original mechanism based on basics of nano-photonics, to modulate speckle patterns.

Recently, we have been developing a zinc-oxide spatial light modulator ( $\mathrm{ZnO}$-SLM) based on techniques that we developed [26]. In experimental demonstrations using our SLM, we obtained extremely large amounts of polarization rotation. In the work described in this paper, to apply this SLM to high-speed speckle modulation, we quantitatively verified the performance of the SLM as an optical switching device. A nitrogen-doped $\mathrm{ZnO}$ single crystal that we fabricated reveals a large magnetic susceptibility, and based on this, we expected that it would be possible to demonstrate a novel technique for modulating the refractive index, as well as a corresponding technique for achieving optical switching, which have been required as one of the methods for the speckle modulation. Based on this concept, a quantitative evaluation of textcolorredspeckle modulation is also described.

\section{Device Fabrication}

\subsection{Optical Annealing}

$\mathrm{ZnO}$ is one of the most widely used direct-transition-type semiconductors due to its wide band gap, high electron mobility, and high transparency to visible light [27]. Although $\mathrm{ZnO}$ exhibits such useful properties, it is technically difficult to implement electrically induced optical functions. One of the major reasons for such difficulty is due to the difficulty in fabricating devices. Generally, in order to realize an electrooptical device by using $\mathrm{ZnO}$ crystal, it is necessary to add p-type dopants to the n-type crystal, so as to fabricate a p-n junction, which exhibits various electro-optical properties, such as a rectifying property, the photovoltaic effect, and so on [28]-[32]. However, in the case of the $\mathrm{ZnO}$ crystal, the sequential generation of donor dopants occurs in the crystal in association with the introduction of acceptor dopants, and they compensate each other [27]. Inevitably, it is technically difficult to fabricate a $\mathrm{p}-\mathrm{n}$ junction having sufficient quality to be used as a practical electro-optical device.

Recently, a technique known as optical annealing [33] has been proposed by the authors' group. The basic setup and mechanism of the method are schematically shown in Fig. 1. 
In the method, in addition to thermal annealing by Joule heating due to the application of a forward bias current between the surface side and underside electrodes, optical energy is also radiated. The base material must be transparent to the optical energy, so that the optical energy can efficiently interact with dopants in the material. In this method, the thermal mobility of dopants is much smaller than in the case of standard thermal annealing using electric furnaces, and the optically-irradiated dopants behave as free radicals. Generally, these unpaired electrons make the free radicals have high chemical-reactivity towards other substances. Especially in the case of optical annealing, this results in more active substitution of dopants with existing substances in the doped material, as shown in Fig. 1(b). Moreover, as our colleagues have previously verified [34], the substituted dopants are not randomly distributed but reveal cluster-like distributions. Based on the fundamentals of molecular chemistry, such materials are expected to reveal larger magnetic susceptibility, and this is the most critical and fundamental point in the following discussions. By utilizing this method, various electro-optical functions have been successfully demonstrated by using several types of semiconductors [35]-[43].

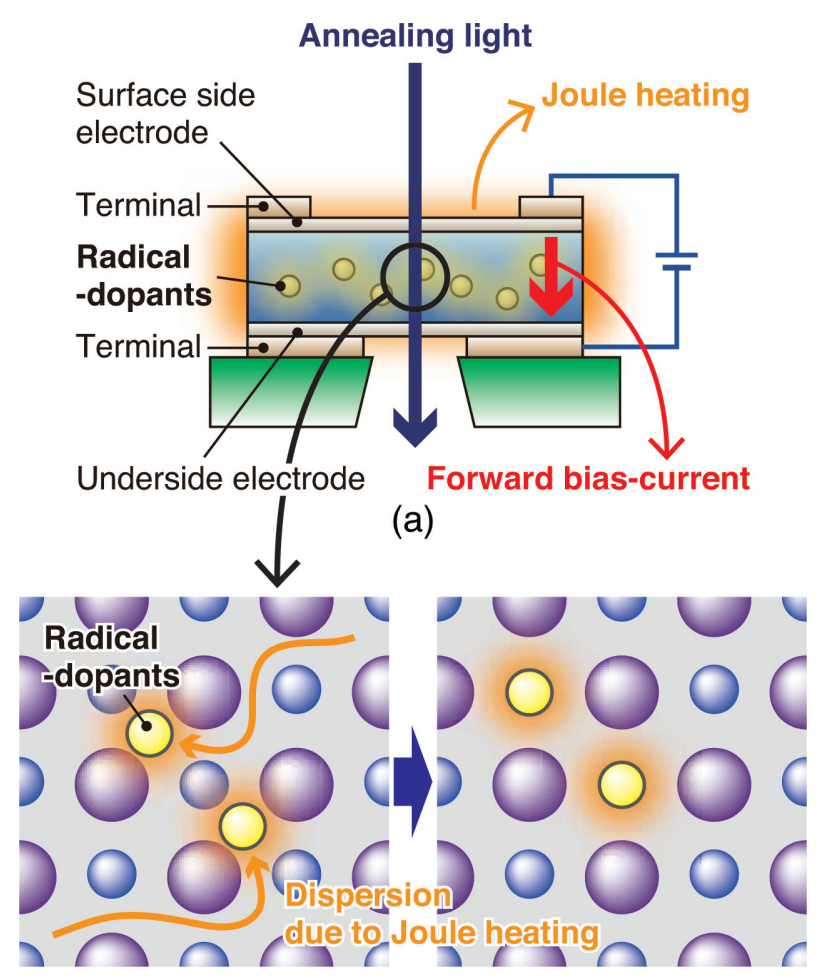

(b)

Fig. 1 Schematic diagram of (a) optical annealing, and (b) the behavior of radical dopants during the process.

\subsection{In-Plane Driving Current}

One of the most familiar physical manifestations of the magneto-optical effect Faraday rotation, which is rotation of the polarization of linearly polarized incident light during propagation in a magneto-optical material by applying an external magnetic field. In such a case, a quantitative evaluation of the magneto-optical effect is described by the Verdet constant $V=\theta \cdot \chi / M \cdot l$, where $\theta, l, M$ and $\chi$ represent the Faraday rotation angle, the length of the light path in the material, the intensity of the magnetization vector, and the magnetic susceptibility, respectively. As shown, the magnetic susceptibility $\chi$ is directly related to the intensity of the magneto-optical effect.

Recently, we have proposed an electro-optical device with a novel structure, which reveals characteristic functions by applying a current in the in-plane direction [26]. Figure 2 shows a schematic diagram of the driving mechanism of the device. As shown, the most important aspect of the idea is the use of current-induced magnetic fields.

As described in the previous section, because the magnetic susceptibility $\chi$ of the doped material is improved by the optical annealing, current-induced magnetic fields can be expected to induce sufficient levels of interactions between the materials and a corresponding level of the magneto-optical effect on incident light. While common experiments on the magneto-optical effect require a large setup to apply a strong magnetic field to the target material, an in-plane current and the resulting current-induced magnetic fields can be achieved with a quite compact setup, as shown in Fig. 2. In particular, because the intensity of the current-induced magnetic field at a point is inversely proportional to the distance between the current and the point, a sufficient level of the magneto-optical effect can be induced only at regions close to the surface of the device, less than $5 \mu \mathrm{m}$ in our setup. Indeed, our group has experimentally demonstrated large amounts of polarization rotation by using current-induced magnetic fields with a setup similar to the one described in this paper [26].

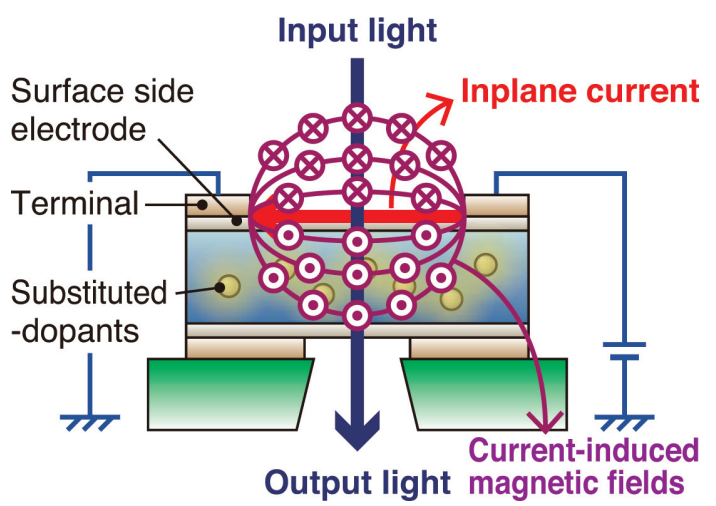

Fig. 2 Schematic diagram of novel electro-optical device driven by in-plane current. 


\subsection{Device Configuration}

Our previous achievements that revealed giant polarization rotation were demonstrated by using a reflective-type device [26]. In order to improve the practicality of our idea, we newly developed a transmissive-type device. Similar to the previous device, a commercially available $\mathrm{ZnO}$ single crystal, which was prepared by the hydrothermal growth method, was used, and nitrogen ions were doped into the crystal by using multi-step implantation [36]. The implantation is done by sequentially applying $\mathrm{N}^{+}$and $\mathrm{N}^{2+}$ with acceleration energies in six steps from $20 \mathrm{keV}$ to $600 \mathrm{keV}$. As a result, the thickness of the doped layer in the $\mathrm{ZnO}$ crystal became $2 \sim 3 \mu \mathrm{m}$ with a dopant density of $10^{18} \sim 10^{19}$ atoms $/ \mathrm{cm}^{3}$, and the depth from the surface of the device was less than $1 \mu \mathrm{m}$. As the surface side and underside electrodes, $150 \mathrm{~nm}$-thick indium tin oxide (ITO) films were deposited by radio frequency (RF) sputtering, and Au terminals were fabricated at each side by deposition and etching processes. Then, the doped-ZnO was set on a glass epoxy substrate, and an aperture was formed to allow transmission of the incident light.

The surface side and underside electrodes were connected so as to perform optical annealing. During 16 hours of annealing, a forward bias current with a current density of less than $0.1 \mathrm{~A} / \mathrm{cm}^{2}$ was applied between the surface side and underside electrodes, which was the highest current that could be applied without causing any thermal destruction of the Au terminals. The photon energy of the annealing light was set at $3.05 \mathrm{eV}$, which is much lower than the bandgap energy of $\mathrm{ZnO}(3.40 \mathrm{eV})$, and the power density of the annealing light focused on the device was $1 \mathrm{~W} / \mathrm{cm}^{2}$.

After the annealing process, the electrode connection was changed to apply the in-plane current for driving the device, as shown in Fig. 3 (a). Here, the same light source as that used for the annealing light was used as the input light source, and it is linearly-polarized by Glan-Thompson polarizer. Due to phase shifts during the propagation in the device, transmitted light reveals elliptical polarization. Photographs of the device are shown in Fig. 3 (b). As shown, four independent units were prepared on a single device to verify crosstalk between each unit. Each unit has four Au terminals, and the in-plane current was applied by connecting two arbitrary terminals of the four terminals.

\section{Refractive Index Modulation}

Polarization rotation based on the magneto-optical effect is one aspect of the circular-polarization selection rule toward optical transitions in a material with high magnetization. In other words, a difference in refractive index between leftand right-handed circularly polarized light corresponds to rotation of linearly-polarized light. Although giant polarization rotation has been previously demonstrated by our group [26], here we focus on modulation of the refractive index and a corresponding beam shift of plane-wave light

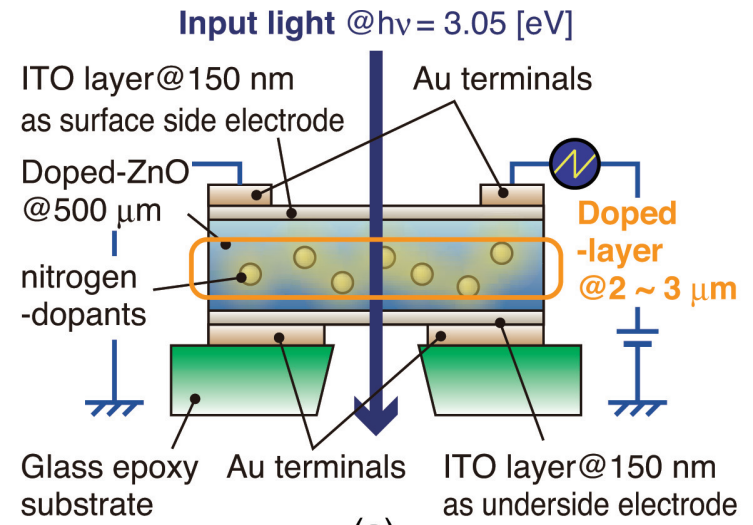

(a)

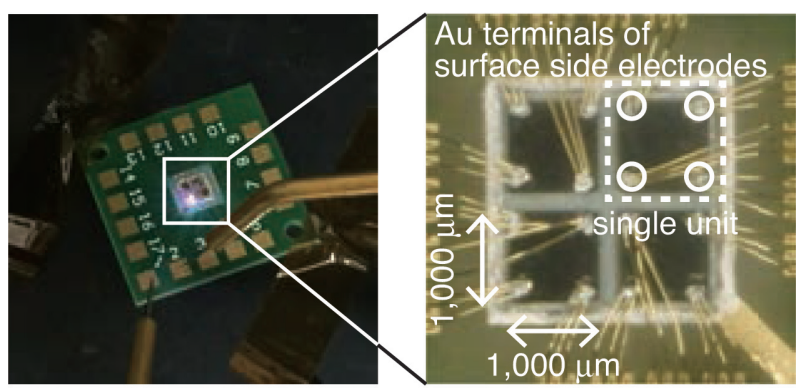

(b)

Fig. 3 (a) Schematic diagram of transmissive-type ZnO SLM and (b) photograph of the device consisting of $2 \times 2$ units.

by using a $\mathrm{ZnO}$-SLM. The basics of the demonstration are described in the following.

As schematically shown in Fig. 4, because a spatial distribution of in-plane current density and a corresponding magnetic field distribution can be made to occur by using two terminals on the surface side electrodes of the SLM, a corresponding spatial distribution of the refractive index of $\mathrm{ZnO}$ is obtained. In such a case, incident plane-wave light is refracted by the SLM. If different pairs of terminals are used, refraction in different directions is induced.

Figure 5 show the results of some demonstrations of the refraction of plane-wave light, where the output light was captured by a CCD camera when voltages of $0 \mathrm{~V}$ (left) and $18 \mathrm{~V}$ (right) were applied to the SLM. In the experiment, only one of the four units was driven, and only the corresponding light, namely, the upper-right light in Fig. 5, was modulated. As shown in Figs. 5 (a) and (b), only the upper-right light was shifted in the vertical and horizontal directions, respectively.

As we have shown, the refraction direction corresponds to the position of the terminals used for injection of the inplane current. As a result, we verified that the spatial distributions of the in-plane current density and the corresponding magnetic fields induced a spatial distribution of the refractive index in the $\mathrm{ZnO}$. During the demonstration shown in Fig. 5 (a), the change in the refractive index, which is approximately calculated from the amount of shift of the in- 


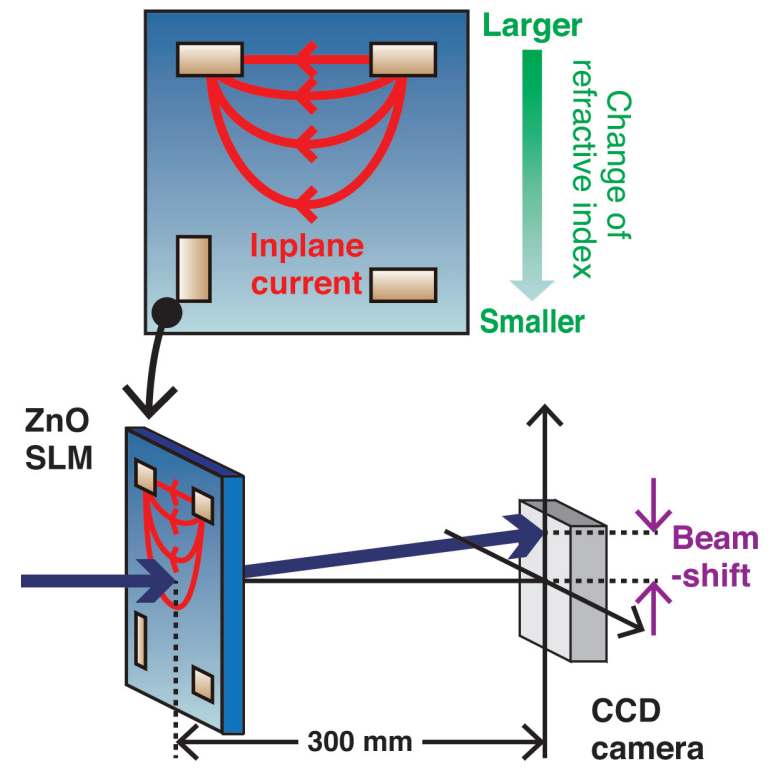

Fig. 4 Schematic diagram of spatial distribution of refractive index due to spatial distribution of in-plane current density (upper), and corresponding beam shift of incident plane-wave light (lower).

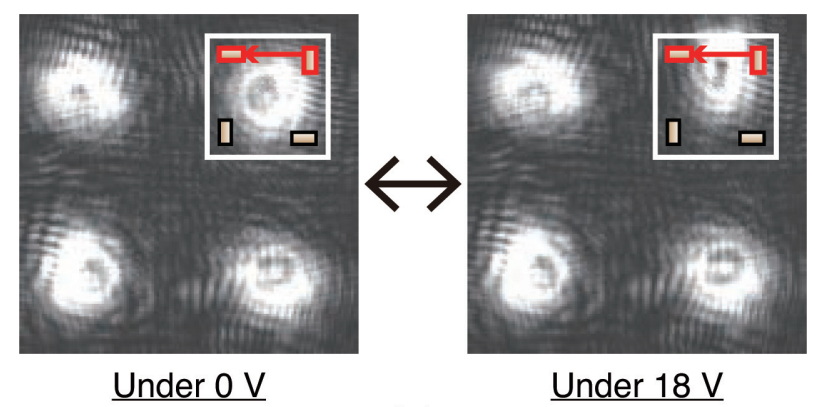

(a)

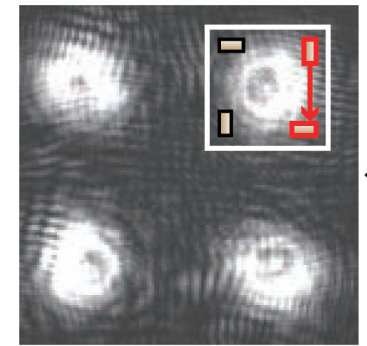

Under $0 \mathrm{~V}$

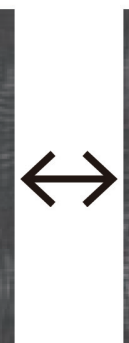

(b)

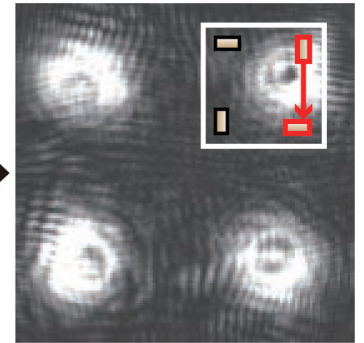

Under $18 \mathrm{~V}$
Fig. 5 Experimental results of refraction of plane-wave light in (a) vertical direction and (b) horizontal direction.

cident light, depends on the voltage applied to the SLM, as shown in Fig. 6.

As shown, a change in refractive index of up to 0.40 , which is $5 \%$ of the change rate relative to the default refractive index of $\mathrm{ZnO}$ crystal (2.00) at room temperature, was obtained by applying a voltage of $20 \mathrm{~V}$. This change is extremely large as compared to that in well-known magneto-

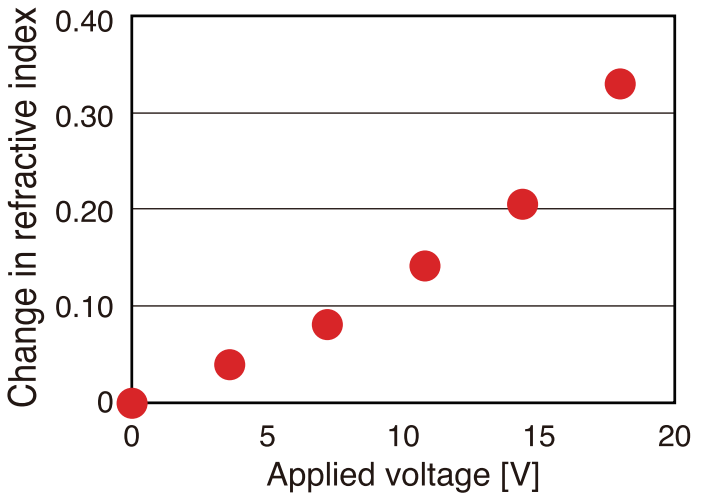

Fig. 6 Change in refractive index.

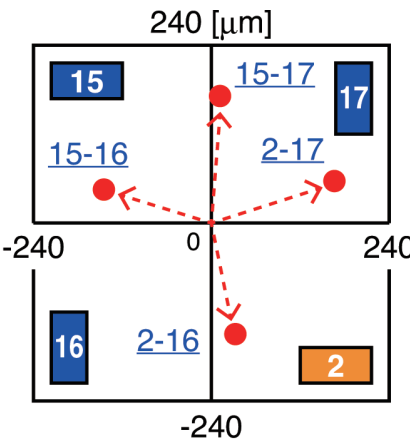

(a)

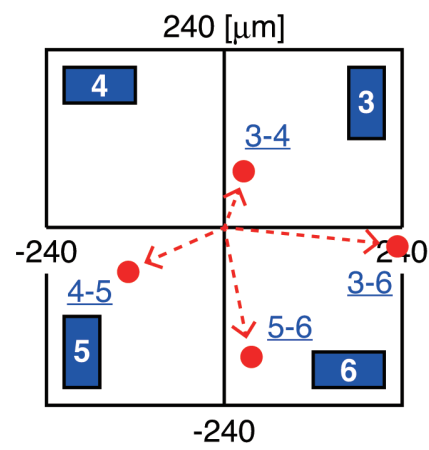

(c)

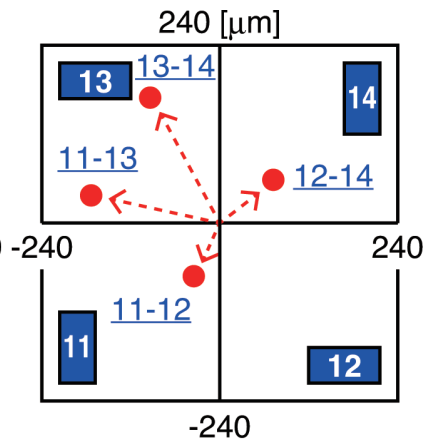

(b)

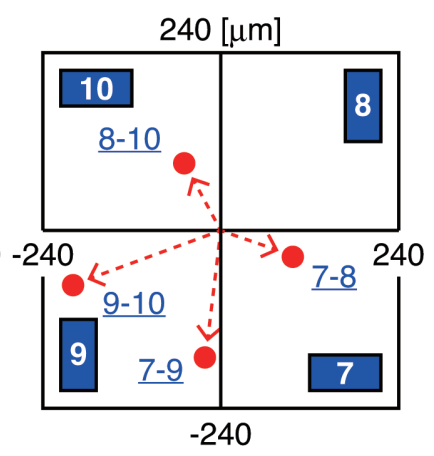

(d)
Fig. 7 Directions and amounts of refraction by using various pairs of terminals with (a) annealed unit and (b), (c), (d) non-annealed units. Terminal 2 is used during the optical annealing process.

optical materials, such as yttrium iron garnet (YIG) [44].

Moreover, various directions and amounts of refraction obtained by using various pairs of terminals are shown in Fig. 7. Figure 7(a) shows a case where an annealed unit is used, and Figs. 7 (b), (c), and (d) shows cases where nonannealed units are used. The squares and number in each square represent the positions and serial numbers identifying terminals of the surface side electrode, respectively, and the underlined numbers represent the used pairs of terminals.

As shown, the amounts of shift and the shift directions with the non-annealed units were more non-uniform, 


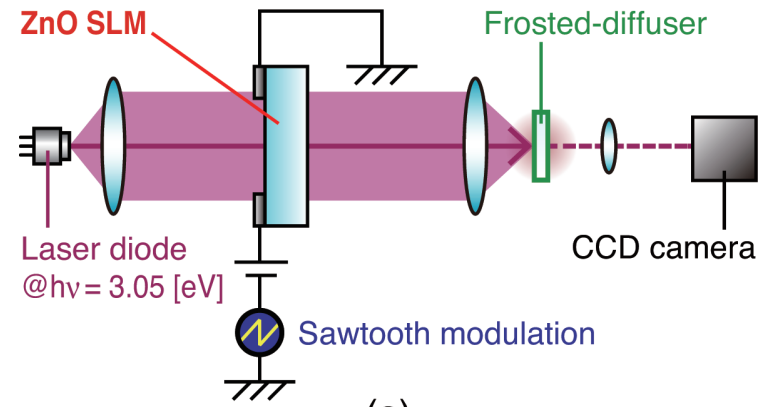

(a)

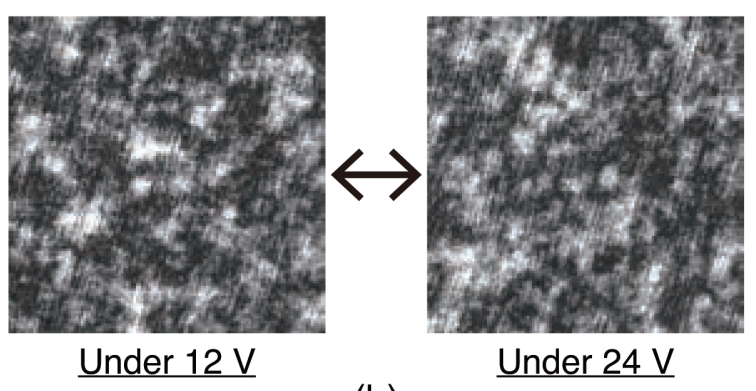

(b)

Fig. 8 (a) Schematic diagram of optical setup for experimental demonstration of speckle modulation, and (b) the results of speckle modulation.

whereas more uniform refraction was obtained with the annealed unit. The results indicate that optical annealing realizes homogeneously-distributed dopants, so that the spatial distribution of the refractive index is homogeneously modulated by using any pairs of terminals.

\section{Speckle Modulation}

We experimentally demonstrated the speckle modulation base on the beam shifts. Because speckle patterns are particular to the surface roughness at each focal point on a target, beam shifts due to the spatial distribution of the refractive index induce various speckle patterns. By modulating the speckle patterns at sufficiently high speed, the contrast of a time-averaged image of the patterns is lower than that of individual patterns.

Figure 8 (a) shows the experimental setup used for demonstrating speckle modulation. Similarly to the previous demonstrations, plane-wave light from a laser source is input to the SLM and is focused on a frosted diffuser to generate speckle patterns. The surface roughness parameter of the diffuser is $\sharp 1,000$.

The focused point of light is shifted by modulation with the SLM, and the speckle patterns generated by the diffuser are correspondingly modulated. The speckle patterns obtained by the CCD camera are shown in Fig. 8 (b). The voltage applied to the SLM as a modulation signal was a sawtooth wave varying from $12 \mathrm{~V}$ to $24 \mathrm{~V}$. A bias voltage of 12 $\mathrm{V}$ was applied so as to use the linear region of the modulation characteristic shown in Fig. 6.

For quantitative evaluation of our method, the speckle

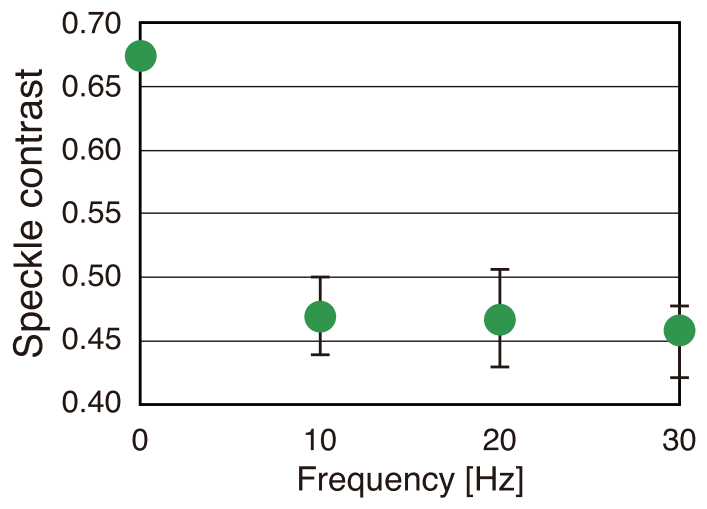

Fig. 9 Reduction of speckle contrast by using ZnO-SLM.

contrast is calculated from trimmed images obtained from video captured by the CCD camera. Here, we obtained a $30 \mathrm{~ms}$ time-averaged image, which can be observed by the human eye. The result is shown in Fig. 9.

As shown, the speckle contrast was successfully reduced from 0.67 to 0.46 . However, the amounts seem to converge to a constant value in the result. This is considered to be due to the polarization selectivity of the magnetooptical effect of the SLM. Namely, because only one direction of circularly polarized light was modulated by the SLM and the other was not, unmodulated speckle patterns remained in the time-averaged image. Thus, speckle contrast cannot be reduced to a sufficient value required for actual use as speckle reduction device, namely, less than 0.10. The result indicates that polarization control of the laser source is important for realizing speckle reduction with our SLM.

As a matter of fact, the results in Fig. 9 only indicates relative changes of speckle patterns induced by our specific setup. As further studies, in order to more strictly discuss the speckle reduction, adopting more general methods for the measurement of speckle contrast must be required [45], [46].

\section{Conclusion}

As shown, we described the development of a specially designed SLM, namely, a transmissive-type ZnO-SLM. Similarly to the authors' previous approach [26], the SLM is fabricated by using optical annealing and is driven by applying an in-plane current, which induces magnetic fields. The results of experimental demonstrations revealed large modulation of the spatial distribution of the refractive index. Moreover, for the future application to the speckle reduction, we focused on high-speed modulation of speckle patterns by utilizing beam shifts of incident plane-wave light due to modulation of the spatial distribution of the refractive index. While the conventional approaches [11]-[25] require a mechanical setup to realize high-speed fluctuation of the displayed screen, the main advantage of our idea is the high speed achieved by a compact setup that does not require any mechanical elements, which lower the stability and durabil- 
ity of the conventional set-ups.

Moreover, the basic mechanism of our method is the magneto-optical effect brought about by current-induced magnetic fields. As is well-known, the basic geometry of the magneto-optical effect is defined by spin flips of electrons in a nanometric space, which are induced in processing times of less than ps-order. Therefore, a compact, high-speed system for speckle reduction can be implemented. In future research, we are planning to quantitatively verify the response characteristics of the modulation and optimize the specifications of the device so that it can be applied to practical speckle reduction for laser displays in the near future.

This work was supported by a research grant from The Murata Science Foundation and by Kyushu University Interdisciplinary Programs in Education and Projects in Research Development.

\section{References}

[1] J.W. Goodman, Speckle Phenomena in Optics, W.H. Freeman, 2010.

[2] N. George and A. Jain, "Speckle reduction using multiple tones of illumination," Appl. Opt., vol.12, no.6, pp.1202-1212, 1973.

[3] C. Saloma, S. Kawata, and S. Minami, "Laser-diode microscope that generates weakly speckled images," Opt. Lett., vol.15, no.4, pp.203-205, 1990.

[4] B. Dingel and S. Kawata, "Laser-diode microscope with fiber illumination," Opt. Comm., vol.93, no.1-2, pp.27-32, 1992.

[5] B. Dingel and S. Kawata, "Speckle-free image in a laser-diode microscope by using the optical feedback effect," Opt. Lett., vol.18, no.7, pp.549-551, 1993.

[6] A. Furukawa, N. Ohse, Y. Sato, D. Imanishi, K. Wakabayashi, S. Itoh, K. Takamura, and S. Hirata, "Effective speckle reduction in laser projection display," Proc. SPIE, vol.6911, pp.69110T-1-7, 2008.

[7] F. Riechert, G. Craggs, Y. Meuret, B. van Giel, H. Thienpont, U. Lemmer, and G. Verschaffelt, "Low-speckle laser projection with a broad-area vertical-cabity surface-emitting laser in the nonmodal emission regime," Appl. Opt., vol.48, no.4, pp.792-798, 2009.

[8] H. Murata, K. Furushoi, Z. Yamamoto, and Y. Okamura, "New speckle control technique using high-speed electro-optic modulators with resonant electrode and polarization-reversed structures," Opt. Rev., vol.19, no.6, pp.436-439, 2012.

[9] J.G. Manni and J.W. Goodman, "Versatile method for achieving $1 \%$ speckle contrast in large-venue laser projection displays using a stationary multimode optical fiber," Opt. Exp., vol.20, no.10, pp.11288-11315, 2012.

[10] H. Murata, K. Shibasaki, Z. Yamamoto, and Y. Okamura, "Speckle control using high-frequency signal superposition to semiconductor laser," Opt. Rev., vol.21, no.1, pp.79-82, 2014.

[11] S. Lowenthal and D. Joyeux, "Speckle removal by slowly moving diffuser associated with a motionless diffuser," J. Opt. Soc. Am., vol.61, no.7, pp.847-851, 1971.

[12] E.G. Rawson, A.B. Nafarrate, R.E. Norton, and J.W. Goodman, "Speckle-free rear-projection screen using two close screens in slow relative motion," J. Opt. Soc. Am., vol.66, no.11, pp.1290-1294, 1976.

[13] H. Amber, Y. Aoki, N. Takai, and T. Asakura, "Mechanism of speckle reduction in laser-microscope-images using a rotating optical fiber," Appl. Phys. B, vol.38, no.1, pp.71-78, 1985.

[14] T. Yoshimura and K. Fujiwara, "Statistical properties of doubly scattered image speckle," J. Opt. Soc. Am. A, vol.9, no.1, pp.91-95, 1992.

[15] L. Wang, T. Tschudi, T. Halldórsson, and P.R. Pétursson, "Speckle reduction in laser projection system by diffractive optical elements,"
Appl. Opt., vol.37, no.10, pp.1770-1775, 1998.

[16] K. Kasazumi, Y. Kitaoka, K. Mizuuchi, and K. Yamamoto, "A practical laser projector with new illumination optics for reduction of speckle noise," Jpn. J. Appl. Phys., vol.43, no.8B, pp.5904-5906, 2004.

[17] S.C. Shin, S.S. Yoo, S.Y. Lee, C.-Y. Park, S.-Y. Park, J.W. Kwon, and S.-G. Lee, "Removal of hot speckle on rear projection screen using the rotating screen system," J. Disp. Tech., vol.2, no.1, pp.79-84, 2006.

[18] I. Fujieda, T. Kosugi, and Y. Inaba, "Speckle noise evaluation and reduction of an edge-lit backlight system utilizing laser diodes and an optical fiber," J. Disp. Tech., vol.5, no.11, pp.414-417, 2009.

[19] S. An, A. Lapchuk, V. Yurlov, J. Song, H. Park, J. Jang, W. Shin, S. Kargapoltsev, and S.K. Yun, "Speckle suppression in laser display using several partially coherent beams," Opt. Exp., vol.17, no.1, pp.92-103, 2009.

[20] Y. Kuratomi, K. Sekiya, H. Satoh, T. Tomiyama, T. Kawakami, B. Katagiri, Y. Suzuki, and T. Uchida, "Speckle reduction mechanism in laser rear projection displays using a small diffuser," J. Opt. Soc. Am. A, vol.27, no.8, pp.1812-1817, 2010.

[21] G. Ouyang, Z. Tong, M.N. Akram, K. Wang, V. Kartashoc, X. Yan, and X. Chen, "Speckle reduction using a motionless diffractive optical element," Opt. Lett., vol.35, no.17, pp.2852-2854, 2010.

[22] M. Kurashige, K. Ishida, T. Takanokura, Y. Ohyagi, and M. Watanabe, "The evaluation of speckle contrast with variable speckle generator,” J. Soc. Inf. Display, vol.19, no.9, pp.631-638, 2011.

[23] Z. Tong, X. Xhen, M.N. Akram, and A. Aksnes, "Compound speckle characterization method and reduction by optical design," J. Disp. Tech., vol.8, no.3, pp.132-137, 2012.

[24] C.-Y. Chen, W.-C. Su, C.-H. Lin, M.-D. Ke, Q.-L. Deng, and K.-Y. Chiu, "Reduction of speckles and distortion in projection system by using a rotating diffuser," Opt. Rev., vol.19, no.6, pp.440-443, 2012.

[25] T.-K.-T. Tran, S. Subramaniam, C.-P. Le, S. Kaur, S. Kalicinski, M. Ekwinska, E. Halvorsen, and M.N. Akram, "Design, modeling, and characterization of a microelectromechanical diffuser device for laser speckle reduction," J. Microelectromech. Sys., vol.23, no.1, pp.117-127, 2014.

[26] N. Tate, T. Kawazoe, W. Nomura, and M. Ohtsu, "Current-induced giant polarization rotation using a $\mathrm{ZnO}$ single crystal doped with nitrogen ions," Scientific Reports, vol.5, Article number 12762, 2015.

[27] Ü. Özgür, Y.I. Alivov, C. Liu, A. Teke, M.A. Reshchikov, S. Doğan, V. Avrutin, S.-J. Cho, and H. Morkoç, "A comprehensive review of $\mathrm{ZnO}$ materials and devices," J. Appl. Phys., vol.98, no.4, pp.041301-1-103, 2005.

[28] N. Yamazoe, "New approaches for improving semiconductor gas sensors," Sens. Actuators B: Chem., vol.5, no.1-4, pp.7-19, 1991.

[29] K.D. Schierbaum, U. Weimar, and W. Göpel, "Comparison of ceramic, thick film, and thin film chemical sensors based upon $\mathrm{SnO}_{2}$," Sens. Actuators B: Chem., vol.7, no.1-3, pp.709-716, 1992.

[30] G. Kiss, Z. Pintér, I.V. Perczel, Z. Sassi, and F. Réti, "Study of oxide semiconductor sensor materials by selected methods," Thin Solid Films, vol.391, no.2, pp.216-223, 2001.

[31] K. Nomura, H. Ohta, A. Takagi, T. Kamiya, M. Hirano, and H. Hosono, "Room-temperature fabrication of transparent flexible thin-film transistors using amorphous oxide semiconductors," Nature, vol.432, no.7016, pp.488-492, 2004.

[32] J.S. Park, W.-J. Maeng, H.-S. Kim, and J.-S. Park, "Review of recent developments in amorphous oxide semiconductor thin-film transistor devices," Thin Solid Films, vol.520, no.6, pp.1679-1693, 2012.

[33] T. Kawazoe, M.A. Mueed, and M. Ohtsu, "Highly efficient and broadband Si homojunction structured near-infrared light emitting diodes based on the phonon-assisted optical near-field process," Appl. Phys. B, vol.104, no.4, pp.747-754, 2011.

[34] T. Kawazoe, K. Nishioka, and M. Ohtsu, "Polarization control of an infrared silicon light-emitting diode by dressed photons and analyses of the spatial distribution of doped Boron atoms," Appl. Phys. A, vol.121, no.4, pp.1409-1415, 2015. 
[35] T. Kawazoe, K. Kobayashi, S. Takubo, and M. Ohtsu, "Nonadiabatic photodissociation process using an optical near field," J. Chem. Phys., vol.122, no.2, pp.024715-1-5, 2005.

[36] K. Kitamura, T. Kawazoe, and M. Ohtsu, "Homojunction-structured $\mathrm{ZnO}$ light-emitting diodes fabricated by dressed-photon assisted annealing,” Appl. Phys. B, vol.107, no.2, pp.293-299, 2012.

[37] T. Kawazoe, M. Ohtsu, K. Akahane, and N. Yamamoto, "Si homojunction structured near-infrared laser based on a phonon-assisted process," Appl. Phys. B, vol.107, no.3, pp.659-663, 2012.

[38] H. Tanaka, T. Kawazoe, and M. Ohtsu, "Increasing Si photodetector photosensitivity in near-infrared region and manifestation of optical amplification by dressed photons," Appl. Phys. B, vol.108, no.1, pp.51-56, 2012.

[39] N. Wada, T. Kawazoe, and M. Ohtsu, "An optical and electrical relaxation oscillator using a Si homojunction structured light emitting diode," Appl. Phys. B, vol.108, no.1, pp.25-29, 2012.

[40] M.A. Tran, T. Kawazoe, and M. Ohtsu, "Fabrication of a bulk silicon $\mathrm{p}-\mathrm{n}$ homojunction-structured light-emitting diode showing visible electroluminescence at room temperature," Appl. Phys. A, vol.115, no.1, pp.105-111, 2014.

[41] N. Wada, M.A. Tran, T. Kawazoe, and M. Ohtsu, "Measurement of multimode coherent phonons in nanometric spaces in a homojunction-structured silicon light emitting diode," Appl. Phys. A, vol.115, no.1, pp.113-118, 2014.

[42] M. Yamaguchi, T. Kawazoe, and M. Ohtsu, "Evaluating the coupling strength of electron-hole pairs and phonons in a $0.9 \mu \mathrm{m}$-wavelength silicon light emitting diode using dressed-photon-phonons," Appl. Phys. A, vol.115, no.1, pp.119-125, 2014.

[43] T. Kawazoe and M. Ohtsu, "Bulk crystal SiC blue LED with p-n homojunction structure fabricated by dressed-photon-phonon-assisted annealing," Appl. Phys. A, vol.115, no.1, pp.127-133, 2014.

[44] S. Geller, H.J. Williams, R.C. Sehrwood and G.P. Espinosa, "Bismuth substitution in Yttrium Iron Aluminum garnets," J. Appl. Phys., vol.35, no.6, pp.1754-1756, 1964.

[45] S. Kubota, "Spatial coherence measurement of a scanning laser system and applicability of the Zernike's approximation to the exit pupil on the scan mirror," Opt. Rev., vol.19, no.6, pp.432-435, 2012.

[46] K. Suzuki, T. Fukui, S. Kubota, and Y. Furukawa, "Verification of speckle contrast measurement interrelation with observation distance,” Opt. Rev., vol.21, no.1, pp.94-97, 2014.

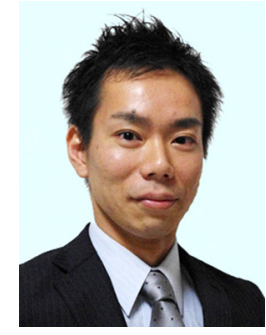

Naoya Tate Dr. degree in information science from Osaka Univ. (2006), Project researcher at the Japan Science and Technology Agency (2006), Project assistant professor (2007) and Project researcher (2011) at the Univ. of Tokyo, Associate professor at Kyushu Univ. (2014).

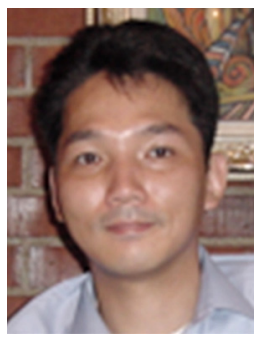

Tadashi Kawazoe Dr. E. degrees in physics from Univ. of Tsukuba (1996), Research associate at Yamagata Univ. (1996), Project researcher at Japan Science and Technology Corporation (2000), Project associated professor (2007) and Project researcher (2011) at the Univ. of Tokyo, General Manager at Nanophotonics Engineering Organization (2015).

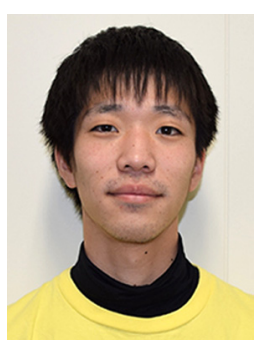

Shunsuke Nakashima $\quad$ B. E. degree in electronics engineering from Kyushu Univ. (2016).

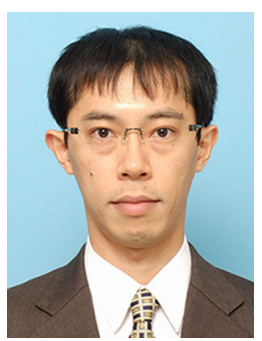

Wataru Nomura Dr. degrees in information science from the Univ. of Tokyo (2007), Project assistant professor (2007) and Project researcher (2011) at the Univ. of Tokyo, Research associate at Kyushu Univ. (2015).

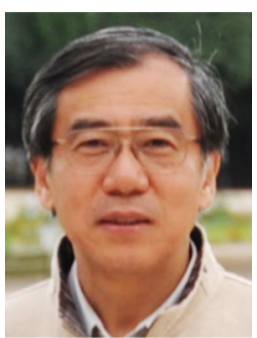

Motoichi Ohtsu Dr. E. degrees in electronics engineering from Tokyo Inst. Tech. (1978), Member of Tech. Staff, AT\&T Bell Labs. (1986-1987), Professor at the Tokyo Inst. Tech. (1991), Professor at the Univ. of Tokyo (2004). Issac Koga Gold Medal of URSI (1984), Japan Royal Medal with a Purple Ribbon from the Japanese Government (2004), Julius Springer Prize for Applied Physics (2009). 